\title{
La vivienda sustentable para abatir el cambio climático. Caso Tepatitlán de Morelos
}

\author{
Sustainable housing to reduce climate change. Tepatitlán de Morelos Case
}

\begin{abstract}
Moradia sustentável para reduzir as mudanças climáticas. Caso Tepatitlán
de Morelos
\end{abstract}

Guillermo José Navarro del Toro

Universidad de Guadalajara, Centro Universitario de los Altos, México

guillermo.ndeltoro@academicos.udg.mx https://orcid.org/0000-0002-4316-879X

\section{Resumen}

Esta investigación se centró en la búsqueda de materiales y tecnologías para la construcción de viviendas de Tepatitlán de Morelos, Jalisco, México, municipio que se está convirtiendo en un polo de desarrollo de viviendas y servicios atractivo para todo tipo y tamaño de empresas. Aprovechando que la Universidad de Guadalajara cuenta con un campus en Tepatitlán, el Centro Universitario de los Altos (CUAltos), se les solicitó a alumnos allí inscritos que llenaran una encuesta que indagaba sobre los materiales con los que se construyeron las casas que habitaban en ese momento. Participaron 80 alumnos de cuatro grupos. Como parte de los resultados se obtuvo que si a las casas habitación se les provee de dispositivos ahorradores de energía, agua y gas se logrará una reducción importante en la huella ecológica, y por lo tanto, se hará una contribución a la sustentabilidad del medio ambiente.

Palabras clave: ecotecnias, medio ambiente, sustentabilidad, tecnologías sustentables. 


\section{Abstract}

This research focused on the search for materials and technologies for the construction of homes in Tepatitlán de Morelos, Jalisco, Mexico, a municipality that is becoming an attractive housing and service development center for all types and sizes of companies. Taking advantage of the fact that the University of Guadalajara has a campus in Tepatitlán, the Centro Universitario de los Altos (CUAltos), students registered there were asked to fill out a survey that inquired about the materials with which the houses that lived in that area were built. moment. 80 students from four groups participated. As part of the results, it was obtained that if the houses are provided with energy, water and gas saving devices, a significant reduction in the ecological footprint will be achieved, and therefore, a contribution will be made to the sustainability of the environment.

Keywords: eco-technologies, environment, sustainability, sustainable technologies.

\section{Resumo}

Esta pesquisa teve como foco a busca de materiais e tecnologias para a construção de casas em Tepatitlán de Morelos, Jalisco, México, um município que está se tornando um pólo de desenvolvimento habitacional e de serviços atraente para empresas de todos os tipos e portes. Aproveitando o fato de que a Universidade de Guadalajara possui um campus em Tepatitlán, o Centro Universitário Los Altos (CUAltos), os alunos ali inscritos foram convidados a responder a uma pesquisa que indagava sobre os materiais com que as casas que moravam naquela área eram construído. momento. Participaram 80 alunos de quatro grupos. Como parte dos resultados, obteve-se que se as habitações forem dotadas de dispositivos economizadores de energia, água e gás, conseguir-se-á uma redução significativa da pegada ecológica e, consequentemente, dar-se-á um contributo para a sustentabilidade do meio ambiente.

Palavras-chave: eco-tecnologias, meio ambiente, sustentabilidade, tecnologias sustentáveis.

Fecha Recepción: Enero 2021

Fecha Aceptación: Junio 2021 


\section{Introducción}

Actualmente, uno de los principales problemas, si no el principal, es el cambio climático. Este fenómeno afecta a todos los países del mundo, sin excepción alguna. Si bien no se pueden descartar del todo ciertas causas naturales, el cambio climático es principalmente resultado de la actividad humana. Tal y como lo plantea el Intergovernmental Panel on Climate Change (IPCC) (Core Writing Team, Pachauri y Meyer, 2014):

La influencia humana en el sistema climático es clara, y las emisiones antropógenas recientes de gases de efecto invernadero son las más altas de la historia. Los cambios climáticos recientes han tenido impactos generalizados en los sistemas humanos y naturales (p. 2).

Los ambientalistas han designado al calentamiento global como la manifestación más representativa del cambio climático.

El calentamiento en el sistema climático es inequívoco, y desde la década de 1950 muchos de los cambios observados no han tenido precedentes en los últimos decenios a milenios. La atmósfera y el océano se han calentado, los volúmenes de nieve y hielo han disminuido y el nivel del mar se ha elevado (Core Writing Team et al., 2014, p. 2).

En países de América Latina, África y Asia, uno de los factores que influye en el cambio climático está relacionado con el tipo de materiales que se emplean en la construcción de casas-habitación, ya que dichos materiales no permiten el ahorro de energía y agua, o son de origen no renovable. El caso contrario sucede en algunos de los países considerados de primer mundo, en donde se emplean materiales renovables que permiten el ahorro de energía, evitan el flujo de calor hacia el exterior de la vivienda en épocas donde las temperaturas descienden, o bien impiden la entrada de calor a la vivienda en las temporadas calurosas. Y no hay que olvidar que, en muchos lugares, se han incorporado sistemas que convierten la energía solar y eólica en energía eléctrica para alimentar los dispositivos utilizados en hogares e industrias, al igual que sistemas de captación de agua de lluvias.

Hay regiones del planeta en donde se emplean tecnologías de iluminación, de acondicionamiento de climas interiores, de cocina y de calefacción de agua que permiten el ahorro de energía eléctrica. Incluso, gracias a ciertas tecnologías, se sustituye el gas por metano generado a partir de desechos orgánicos producidos en el propio hogar. Igualmente, 


\section{Revista Iberoamericana}

de las Ciencias Sociales y Humanísticas

ISSN: 2395 - 7972

a partir de ciertas implementaciones, se reutiliza el agua de la regadera o del lavamanos para descargar el inodoro.

Podría pensarse que usar dispositivos ahorradores en la casa o empresa es muy caro porque sus costos iniciales así lo muestran, pero, con el paso del tiempo, es posible ver los ahorros monetarios que se logran, sin olvidar que entre menos desperdicios haya más se cuida al medio ambiente.

\section{Marco teórico}

En la vida cotidiana, dentro de nuestros hogares, regularmente hacemos uso excesivo de materiales (insumos o materias primas) no renovables Y no es asunto menor, ya que allí pasamos gran parte del día, un tercio o más de las 24 horas.

El ser humano depende de la energía eléctrica, gas y agua a diario para desarrollar muchas de sus actividades. Por poner un ejemplo, de acuerdo con el Consejo Consultivo del Agua (La Silla Rota, 22 marzo de 2018), el consumo promedio de una persona diario oscila entre 150 y 200 litros. Desgraciadamente, a la mayoría de las personas no les preocupa la forma en que estos recursos llegan hasta sus hogares. Algunos de los procesos de generación son altamente contaminantes. El agua requiere de procesos de saneamiento que regularmente implican energía eléctrica y productos químicos . Otro tipo de contaminación es la generada al quemar gas o extraerlo. Se trata de casos que toman lugar a diario en un sinnúmero de lugares del planeta de manera simultánea.

Por ello, en esta investigación se tratará de exponer algunos de los beneficios ambientales y económicos derivados del uso de tecnologías amigables con el medio ambiente, específicamente en los hogares de la región de Tepatitlán, Jalisco, México. Asimismo, se expondrán las razones por las que se deben de sustituir las tecnologías y materiales que actualmente predominan por unos ambientalmente más responsables. Para lograr este propósito, a los habitantes de la región se les debe concientizar sobre la cantidad de recursos no renovables que una vivienda requiere para su funcionamiento diario, de acuerdo con las demandas de la vida actual. De igual manera, se tiene que presentar el cálculo del costo promedio se requiere para sustentar una vivienda, incluyendo los pagos de energía eléctrica, gas y agua, lo cual debe de adecuarse al modelo de vida de los habitantes del lugar geográfico objeto de estudio. Por otro lado, y como contraparte, se tienen que determinar los medios tecnológicos existentes que ayudan a disminuir el gasto de recursos en una vivienda 


\section{Revista Iberoamericana}

de las Ciencias Sociales y

Humanísticas

ISSN: $2395-7972$

tipo con las características de Tepatitlán. Por último, contrastar los gastos ocasionados con las características actuales en un hogar de la población tepatitlense y los gastos que se tendrían si se emplean materiales y dispositivos ahorradores de energía.

\section{Método}

Se propuso conocer las condiciones actuales de la vivienda promedio en el área de Tepatitlán, Jalisco; las condiciones económicas promedio de sus habitantes, y los materiales más frecuentes empleados en la construcción. De igual manera, se buscan las tecnologías que pueden ser empleadas para las futuras construcciones y los beneficios que presentan en los ámbitos económico y ecológico.

\section{Desarrollo}

El cambio climático es resultado del uso excesivo de prácticas y materiales que perjudican los recursos naturales, contaminan el medio ambiente y alteran el orden natural de todos los seres vivos que habitan el planeta. No es un fenómeno reciente, por el contrario, se viene gestando desde hace años, lo que pasa es que no es hasta apenas unas décadas atrás que el ser humano empezó a adquirir conciencia del daño que le hacía el medio ambiente con la creación y uso de muchos productos de uso constante. Además, no solo son nocivos al momento de crearlos y usarlos, sino que también lo son una vez que han sido utilizados y desechados, ya que a algunos de ellos les toma hasta cientos de años degradarse, tiempo durante el cual continúan contaminando.

También, existe una sobreexplotación de las fuentes de agua dulce. Muchos procesos tanto caseros como industriales se convierten en agentes contaminantes de todo tipo de cuerpo lacustre, a donde el agua regresa después de haber sido usada y contaminada.

Igualmente, existen muchos sistemas de generación de electricidad que implican la quema de combustibles altamente nocivos para la Tierra. A pesar de ello, son los que imperan prácticamente en todo el mundo, ya que hoy en día los sistemas no contaminantes de generación de energía eléctrica no han logrado consolidarse.

De igual manera, en una gran cantidad de hogares se emplea el gas licuado tanto para cocinar como para calentar el agua que se emplea en el baño, cuyas emisiones son una amenaza constante para la capa de ozono. 


\section{Revista Iberoamericana}

de las Ciencias Sociales y

Humanísticas

ISSN: $2395-7972$

Esta es tan solo una pequeña parte de las fuentes de contaminantes que se relacionan con las casas-habitación. Por supuesto, no se han mencionado las fuentes de contaminación provenientes de los parques vehiculares y las industrias, entre otras más.

\section{Datos relevantes de Tepatitlán}

Tepatitlán está catalogada como una ciudad en continuo crecimiento poblacional e industrial que basa su economía en las actividades comerciales, pecuarias y forestales, además de contar con centros educativos que permiten a la gente de las poblaciones vecinas acudir a prepararse en lugar de desplazarse al Área Metropolitana de Guadalajara, lo que ocasiona una mayor demanda de vivienda, aunado a la que se genera por el crecimiento de las empresas regionales que requieren los servicios de personal que proviene de otras latitudes

Como primera parte de este estudio, se consideró que es de vital importancia tomar en consideración las condiciones climáticas que existen en la región, y así determinar los tipos de materiales y tecnologías que pueden ser utilizadas para la construcción en el municipio.

En lo que respecta al clima, es semiseco, con primavera e invierno secos, o semicálido con inviernos benignos. La temperatura media anual oscila entre los $19{ }^{\circ} \mathrm{C}$ y $\operatorname{los} 30.5^{\circ} \mathrm{C}$. Entre los meses de junio, julio y agosto (un promedio de 80 días por año), se tiene una precipitación pluvial media de $874.7 \mathrm{~mm}$. En promedio, hay 18 días con heladas. El resto de los días son soleados con vientos dominantes de sur a norte.

Ahora bien, sus recursos hidrológicos son proporcionados por los ríos Verde y Calderón, del Valle y Tepatitlán. Y cuenta con cuatro presas importantes: Carretas, La Red, Jihuite y El Pantano.

\section{La vivienda actual}

Aprovechando que la Universidad de Guadalajara cuenta con un campus en Tepatitlán, el Centro Universitario de los Altos (CUAltos), se les solicitó a alumnos allí inscritos que llenaran una encuesta que indagaba sobre los materiales con los que se construyeron las casas que habitaban en ese momento. Participaron 80 alumnos de cuatro grupos. Además de su nombre, se les preguntó por su lugar de origen o procedencia. Aquí, 70 de ellos respondieron que provenían de otras poblaciones. 


\section{Revista Iberoamericana \\ de las Ciencias Sociales y Humanísticas}

ISSN: 2395 - 7972

Otra pregunta que se les planteó estuvo relacionada con su hospedaje en Tepatitlán, y resultó que rentan casas (entre varios de ellos para abaratar costos) que son relativamente "modernas" y se encuentran localizadas en los fraccionamientos de reciente creación, por lo que los materiales que se emplean son tabicón de concreto con muros enjarrados, ventanas de vidrio sencillo y puertas de lámina doble. A partir de una inspección visual a la casa de algunos estudiantes que residen en la colina más cercana al CUAltos, se pudo establecer que son casas desarrolladas por compañías constructoras, que son económicas y no están provistas de dispositivos que les permitan tener ahorros en los servicios de eléctrica, gas y agua.

El resto de los estudiantes (10) son nativos de Tepatitlán y viven con sus familias en las denominadas colonias y barrios tradicionales o antiguos. Allí usaron en la construcción ladrillo rojo con enjarres en muros; las ventanas, al igual que las puertas, algunas son metálicas y otras de madera. Están provistas de calentadores de gas. Algunos usan focos ahorradores o de bajo consumo de energía y otros son incandescentes. El sanitario es de seis litros.

\section{Búsqueda de las tecnologías orientadas al revertir el cambio climático}

Como parte de la propia investigación se tuvo que tomar en consideración el significado de algunos de los tecnicismos que se ven involucrados en la protección del medio ambiente. Así, de acuerdo con Calvente (2007), la sustentabilidad se puede definir como la capacidad desarrollada por un sistema para satisfacer las necesidades de las generaciones actuales sin comprometer los recursos y oportunidades para el crecimiento y desarrollo de las generaciones futuras.

Con este principio se partió para buscar los materiales y dispositivos que pudieran ser empleados en la construcción de las nuevas casas-habitación que se pretendan utilizar en los futuros desarrollos que se hagan en Tepatitlán. Igualmente, se tomó en cuenta que se tenía que lograr un balance entre los costos, calidad y protección ambiental, tal y como lo plantea la Organización de las Naciones Unidas [ONU] (1987).

En efecto, uno de los factores que se tomaron en consideración en la selección de las tecnologías a utilizar fue el económico, ya que, de seguir creciendo la población, habrá una mayor demanda de vivienda. Se trata de la oportunidad para establecer un sistema de 


\section{Revista Iberoamericana}

de las Ciencias Sociales y

Humanísticas

ISSN: $2395-7972$

preservación y restablecimiento de la naturaleza, como lo ha mencionado Barnett (23 de noviembre de 2017).

Si a las casas habitación se les provee de dispositivos ahorradores de energía, agua y gas se logrará una reducción importante en la huella ecológica, y por lo tanto, se hará una contribución a la sustentabilidad del medio ambiente, lo que concuerda con lo que explica Garrett (27 de mayo de 2021).

Combinar apropiadamente las tecnologías de ahorro de energía, los materiales de construcción y el cuidado del medio ambiente para revertir el cambio climático es cumplir con las condiciones que se han descrito como propias de una vivienda sustentable. Aún más, si se hace extensivo a los nuevos proyectos de habitación, entonces estaríamos hablando de un nuevo paradigma de construcción para la nueva vivienda (Baeza, s. f.).

\section{Dispositivos y materiales para la reducción de la huella humana para protección del medio ambiente}

La búsqueda de dispositivos que permiten utilizar materiales y dispositivos de ahorro de energía para la protección del medio ambiente con el propósito de contribuir a revertir el cambio climático se relaciona directamente con la denominada arquitectura sustentable, siguiendo aquí a Miceli (2016).

Las ecotecnias que forman parte de la arquitectura sustentable surgieron como resultado del aprovechamiento óptimo de los recursos naturales y los combustibles fósiles. Por ejemplo, los paneles solares fotovoltaicos y los térmicos, los cuales usan como principal fuente la energía solar, son una tecnología que está adquiriendo un gran auge y cuyo uso es un gran paso para revertir el cambio climático (Acciona, 2016; Fehrenbacher, 2016). Por su parte, Fredrick (20 de noviembre de 2017) expone las ventajas de los lavabos de descarga doble: "Además del beneficio medioambiental del ahorro de agua, (...) también reduce[n] tu factura de agua con el tiempo" (párr. 3). Y para evitar el intercambio de energía calorífica de la casa hacia fuera y viceversa están las ventanas de doble vidriado (Ral Aluminio, 2018). 
Revista Iberoamericana

de las Ciencias Sociales y

Humanísticas

ISSN: $2395-7972$

\section{La economía familiar y distribución del gasto}

Todas las poblaciones de México están dentro del catálogo del Instituto Nacional de Estadística y Geografía (Inegi) y la Comisión Nacional para la Protección y Defensa de los Usuarios de Servicios Financieros (Condusef), y la población de Tepatitlán no es la excepción.

Entre los datos que se encontraron en las instituciones gubernamentales a las que se acudió, se pudo establecer que en los rubros de vivienda, ingresos y gastos del hogar, la la región de los Altos donde se encuentra Tepatitlán se coloca en el segundo escalón de ingresos dentro del estado de Jalisco, después de las poblaciones que componen el Área Metropolitana de Guadalajara.

Respecto a los gastos más comunes que tienen las personas que habitan en la zona, se encontró que una familia promedio distribuye sus gastos en el pago de vivienda, agua, luz, gas y otros combustibles, lo que representa $19.8 \%$ del total de las entradas por familia; $25.8 \%$ lo utiliza en alimentos y bebidas no alcohólicas, mientras $12.9 \%$ lo emplea en el transporte (ver figura 1).

Disminuir ese $19.8 \%$ que emplea en vivienda, agua, luz, gas y otros combustibles es una gran oportunidad de ahorro, sobre todo por medio de dispositivos que permiten reducir el consumo de energía eléctrica utilizando tecnologías basadas en la energía solar, o usando las ventanas de doble vidriado, que evitan la pérdida de energía calorífica de dentro de la casa hacia afuera e impiden que las corrientes de aire frío o caliente entren la casa.

Aunado a lo anterior, si a la casa se le provee de un calentador solar, el consumo de gas licuado reducirá y así habrá otra oportunidad de ahorro. Y si todavía se incluye algún otro tipo de inodoro, por ser el que representa mayor consumo de agua en la casa, y se emplea alguno que permita el reciclaje del agua, se estaría cumpliendo el listado de tecnologías alternativas para la casas-habitación marcado por Bohoslavski (1998). 
Revista Iberoamericana

de las Ciencias Sociales y

Humanísticas

ISSN: $2395-7972$

Figura 1. Distribución del dinero del rubro en los que más se gasta en las viviendas

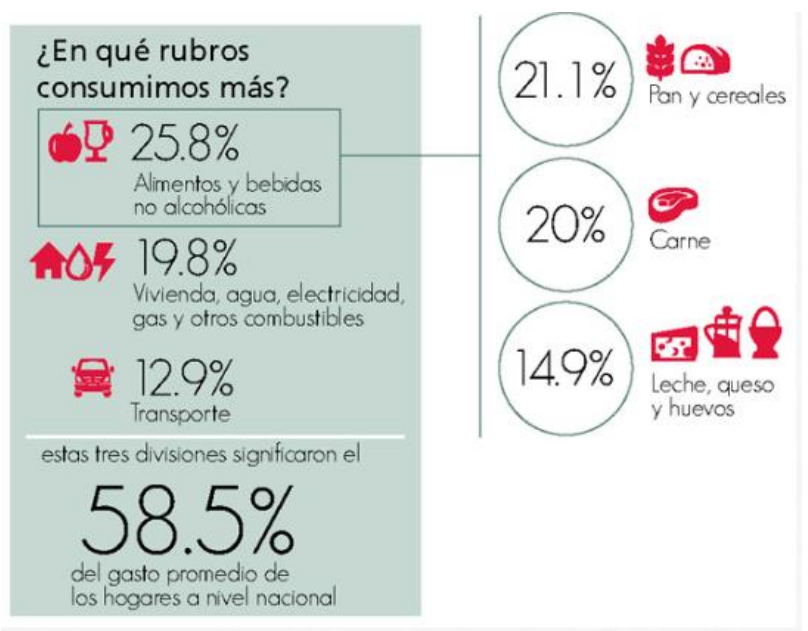

Fuente: Condusef (2021) concepto de distribución del dinero en viviendas, recuperado de Www.condusef.gob.mx

Si bien la aplicación de todas estas implementaciones traerá como consecuencia el ahorro de gastos, el mayor beneficio es que se aporta a la sustentabilidad del planeta y, por ende, a revertir el cambio climático

\section{Resultados}

Para la aplicación de la encuesta de este trabajo se contó con el apoyo de ocho estudiantes; a cada uno se le entregó 30 encuestas para que nos apoyaran aplicándolas entre sus conocidos o vecinos. Sin embargo, uno de los entrevistadores, al tener más vecinos, optó por sacar cuatro copias fotostáticas extras y aplicarlas a cuatro personas más. Ese detalle nos fue comunicado por la persona que capturó los resultados y nos apoyó con las gráficas, por lo cual no hubo ningún inconveniente en dejar las gráficas y resultados tal y como se nos entregaron.

Se acudió a ellos debido a que conocen las zonas en donde habitan, además de que es una gran oportunidad para que la gente de la población en general se vaya adentrando en las tecnologías que se emplean para la sustentabilidad. Como ya lo dijimos, estas no solo representan, en caso de ellos incorporarlas, ahorros económicos, sino que contribuyen al cuidado del medio ambiente de su ciudad.

Para poder determinar qué tanto sabe la gente sobre las viviendas sustentables, así como con el fin de establecer el grado de conocimiento de su casa actual y de una vivienda sustentable, conocer cuánto de su gasto familiar lo emplea en el pago de servicios eléctricos, 
Revista Iberoamericana

de las Ciencias Sociales y

Humanísticas

ISSN: 2395 - 7972

pero sobre todo saber cuánto está dispuesto a invertir en la adquisición de una casa-habitación sustentable, la encuesta consideró cuatro preguntas, las cuales se pueden observar en la figura 2.

Todo ello tuvo como propósito inicial el determinar la urgencia que existe por la construcción de viviendas sustentables, o bien la adaptación de dispositivos ahorradores en sus hogares actuales, así como poder buscar entre las compañías constructoras locales alguna que, en alianza con la universidad y empresarios de la región, esté dispuesta a invertir en revertir el cambio climático.

Figura 2. Encuesta que fue presentada a 244 entrevistados

Pregunta 1. ¿Has escuchado de la vivienda sustentable?

Respuestas : SI NO

Pregunta 2. ¿Tienes idea de lo que representaría para tu hogar vivir en una vivienda sustentable?

Respuestas : SI NO

Pregunta 3. ¿Normalmente a cuánto asciende el gasto de energía eléctrica por mes?

Respuestas: $0 \$ 150 \quad 0 \$ 250 \quad 0 \$ 4000 \$ 500$ ómás

Pregunta 4. ¿Cuánto estarías dispuesto a pagar por una vivenda sustentable?

Respuestas : $\quad 0 \$ 800000 \quad 0 \$ 1^{\prime} 000000 \quad 0 \$ 1^{\prime} 500000$

○ \$2'000 000 o Depediendo qué tan sustentable

Fuente: Elaboración propia

La pregunta número uno fue “¿Has escuchado de la vivienda sustentable?” y las opciones para responder se limitaron a dos opciones, Sí o No (ver figura 3). 
Revista Iberoamericana

de las Ciencias Sociales y Humanísticas

ISSN: $2395-7972$

Figura 3. Conocimiento de viviendas sustentables

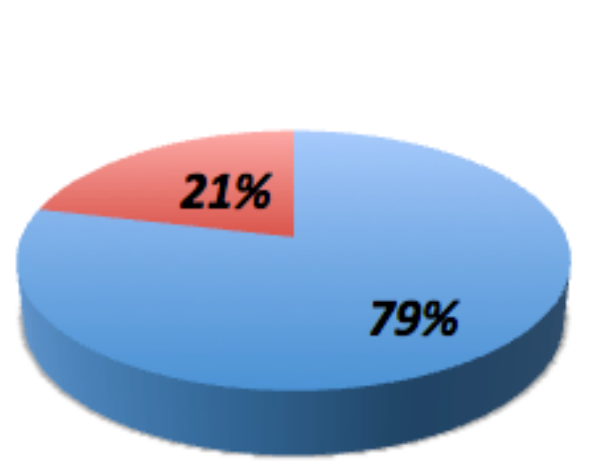

$=\mathrm{SI}$

Fuente: Elaboración propia

El número de personas que contestó afirmativamente fue de 192, lo que representa un alto grado de conocimiento sobre la vivienda sustentable, $79 \%$ de los 244 entrevistados. El porcentaje restante $(21 \%)$ desconoce los beneficios de contar con una vivienda de este tipo.

La pregunta número 2 fue " ¿Tienes idea de lo que representaría para tu hogar vivir en una vivienda sustentable?” y las opciones igualmente se limitaron a un Sí o No (ver figura $4)$.

Figura 4. Ahorro de las viviendas sustentables

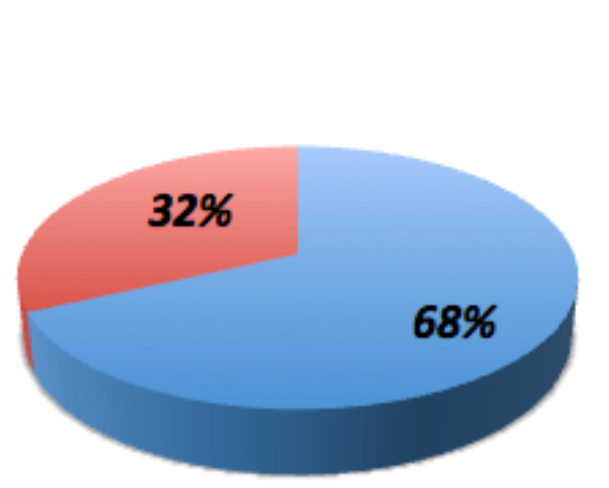

\section{SI}

\section{NO}

Fuente: Elaboración propia

La figura 4 refleja que $68 \%$ de los encuestados, 166 de los 244, sabe de los beneficios que se pueden obtener por el hecho de habitar en una vivienda sustentable, mientras que 78 personas, es decir, $32 \%$ de las respuestas, aún no han visitado alguna vivienda sustentable ni tampoco han tenido ninguna acercamiento con alguna persona a la que le pudieran preguntar sobre la diferencia entre vivir en una casa normal y una sustentable. 
Revista Iberoamericana

de las Ciencias Sociales y Humanísticas

ISSN: $2395-7972$

En la pregunta tres, “¿Normalmente a cuánto asciende el gasto mensual de energía eléctrica por mes?", las opciones que se presentaron fueron: $\$ 150, \$ 250, \$ 400$ y $\$ 500$ o más (ver figura 5).

Figura 5. Gasto mensual de energía eléctrica

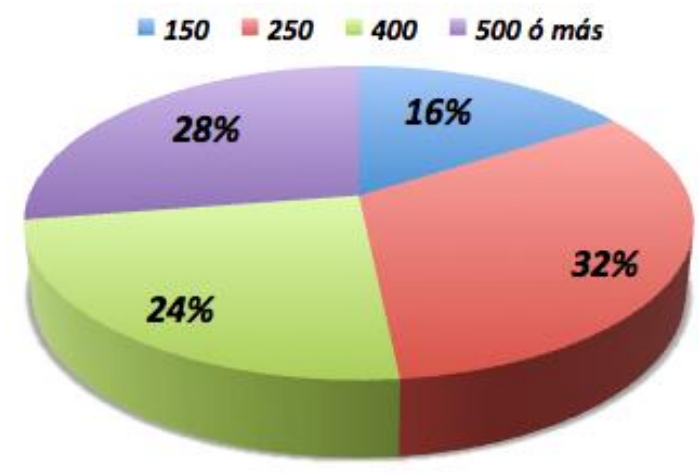

Fuente: Elaboración propia

En cuanto al gasto mensual del servicio de energía eléctrica, el resultado indica que solamente 67 entrevistados de 244 pagan $\$ 500$ pesos ó más, lo que equivale a $28 \%$, mientras que 59 entrevistados, es decir, $24 \%$, pagan $\$ 400$ mensuales por el servicio de energía eléctrica. Por su parte, 79 entrevistados (32\%) pesos manifestaron realizar un pago mensual de servicio de energía eléctrica por $\$ 250$ pesos y solamente 39 entrevistados (16\%) pagan el servicio de energía eléctrica de $\$ 150$ pesos. Por lo anterior, se tiene una oportunidad para las viviendas sustentables, por el ahorro que significa pagar por ese servicio menos dinero.

El cuarto y último ítem consistió en la pregunta “¿Cuánto estarías dispuesto a pagar por una vivienda sustentable?". Las posibles respuestas que se plantearon estuvieron en los costos promedio de las viviendas actuales (ver figura 6). 
Figura 6. Disposición de los habitantes al pago económico

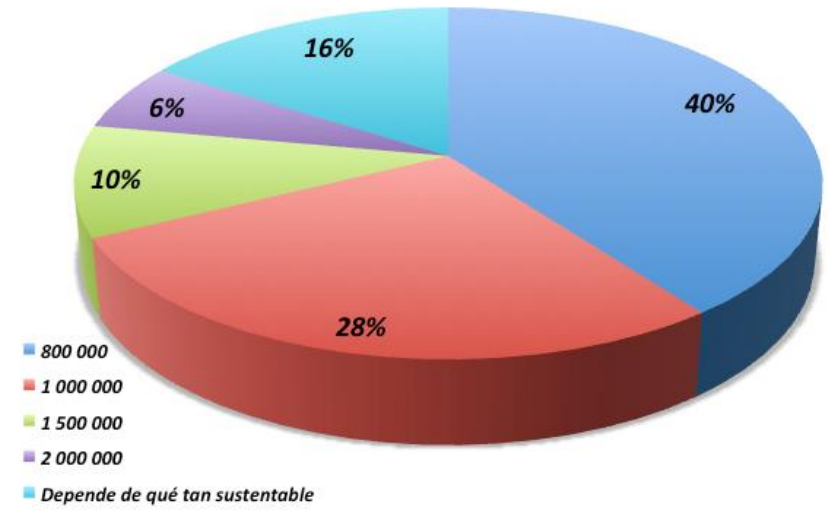

Fuente: Elaboración propia

Así, $40 \%$ de los encuestados está dispuesto a pagar 800000 pesos por una de las viviendas; $28 \%$ de los encuestados está dispuesto a pagar 1000000 de pesos; $16 \%$ de los entrevistados pagaría dependiendo del grado sustentabilidad que tuviese la vivienda; $10 \%$ estaría dispuesto a pagar 1500000 pesos por una vivienda sustentable, y solamente $6 \%$ pagaría los 2000000 de pesos. Un hecho remarcable es que 179 entrevistados mencionaron que sería muy bueno que se construyeran viviendas a manera de coto en las inmediaciones del CUAltos, debido a que la mayoría de las familias de Tepatitlán de Morelos tienen hijos o hijas que actualmente están cursando alguna carrera allí, o lo harán en el futuro, por lo que su cercanía sería un motivo más para participar en la adquisición de una vivienda sustentable.

Finalmente, se puede decir que CUAltos está construido en lo que podría decirse la parte más nueva de Tepatitlán, queda a 15 minutos de lo que es la plaza central; cuenta con servicios de transporte y comunicación aceptables, además de que, por haberse construido en lo que fuera una zona de orientación agrícola, cuenta con agua y espacios sin construir que poco a poco se les ha ido dotando de servicios como son energía eléctrica y derivaciones del agua potable. Por lo que podrían ser tomados en consideración por los urbanizadores como primera posibilidad para la implementación de viviendas sustentables en la región.

\section{Análisis de los resultados de la investigación}

A partir de los datos obtenidos se puede apreciar que es extremadamente urgente iniciar con los desarrollos de colonias sustentables, ya que es una zona con una gran expansión de habitantes. 


\section{Revista Iberoamericana \\ de las Ciencias Sociales y Humanísticas}

ISSN: $2395-7972$

Eso reside en el fenómeno de que las denominadas familias tradicionales de la región se componían de 10 o más habitantes, cuyos descendientes buscan o buscarán en un futuro cercano una vivienda propia, por un lado. Y por el otro, la creación de nuevas empresas en la región o el crecimiento de la ya existentes ha obligado a que lleguen personas de otras poblaciones y demanden viviendas, al ser personas que son profesionistas, muchos de ellos trabajan para la universidad y los distintos centros educativos de la región, pues están acostumbrados a la comodidad y están dispuestos a pagar por ello.

Además, por ser una población que cuenta con empresas de talla internacional, se cuida mucho de que se implementen medidas que ayuden a paliar el cambio climático, no solo dentro de ellas, sino en toda la comunidad.

Ahora bien, de acuerdo con las estadísticas de desarrollo de la población, la inversión que se hace por parte de autoridades y empresas con continuar con una población de tipo tradicional, como lo es Tepatitlán, que originalmente tenía una vocación principalmente pecuaria, sufre una metamorfosis con la llegada del CUAltos casi a finales del siglo pasado, pues vino a impulsar las mejoras de las empresas de la región, que a su vez están impulsando programas para que sus colaboradores y familias se logren preparar en las diversas especialidades que se ofrecen.

Todos, de manera conjunta, empresas, universidad y autoridades municipales, están dispuestos a poner su granito de arena para la generación de sistemas que puedan ayudar o contribuir a revertir el cambio climático. Es el momento de iniciar a utilizar dispositivos ahorradores en las casas-habitación existentes, lo que se podría hacer con las facilidades que brinda el municipio para la obtención de calentadores solares e iniciar con la instalación de sistemas de generación de energía eléctrica en las casas-habitación con el apoyo de las empresas, ya que ellas fueron las primeras que los colocaron en sus instalaciones. 


\section{Discusión}

Como se ha podido ver a través del presente documento, existe una gran urgencia que dar inicio con programas municipales, estatales, federales y privados, combinados preferentemente, para que la nueva cultura del ahorro de energías se adentre entre los pobladores de Tepatitlán. La gente estará dispuesta a participar por el simple hecho de que se le diga que podrá tener ahorros económicos y que redundará en beneficio de su familia, pero sobre todo porque contribuirán a revertir los efectos del cambio climático que están afectando no solo a ellos, sino a todo el planeta.

\section{Conclusiones}

La gente de cualquier región, durante años, no tuvo conciencia sobre el daño que se le hacía al medio ambiente al encender un foco, al calentar el agua en su casa o al encender el ventilador o el clima artificial que tenía en su casa para que estuviera en mejores condiciones.

Aunque nuestro país firmó los tratados que están orientados a disminuir los efectos del cambio climático, tal parece ser que la información nunca llegó a la población en general, aunque se haya anunciado por parte de las autoridades federales, aun cuando se hayan abierto oficinas dedicadas a atender esos problemas. Curiosamente, aquellas personas que llegaron con la universidad fueron las primeras en dar a conocer lo que estaba sucediendo en el mundo, y tal parece ser que aún así a poca gente le importó, por ello es que en este documento se buscó la forma de que la gente se interesara por los problemas del medio ambiente sin mencionarles todas las teorías, sin explicarles todos los elementos que las componen ni la forma en que interactúan; simplemente se buscó llamar su atención de la forma más sutil posible, y se pudo encontrar que la forma más sencilla es hablarles del ahorro que podrán obtener por usar o dejar de usar algo.

Cuando la gente vea que va a poder contar con más dinero en su bolsillo, debido a que ha dejado de pagar algunos servicios o pagará menos por ellos, se involucrarán sin reservas en los programas que implementan la incorporación de las tecnologías sustentables. 
Revista lberoamericana

de las Ciencias Sociales y Humanísticas

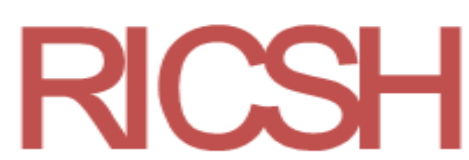

ISSN: 2395 - 7972

\section{Trabajo futuro}

Como integrantes del CUAltos se tiene la obligación moral de participar en los programas que ayuden a mitigar los efectos del cambio climático, pero también es indispensable que se haga extensiva esta participación a los propios estudiantes, y que ellos a su vez involucren a sus familiares y amigos.

Por tal motivo, queda pendiente proponer ante las autoridades universitarias un programa que motive a los estudiantes por medio de la obtención de créditos a que se involucren en actividades ambientales. El cambio climático es un problema muy serio a nivel mundial, por lo que cualquier pequeña aportación que puedan hacer los estudiantes como parte de esos programas, por más pequeño que sea, será benéfico para el planeta.

Aprovechando la experiencia que tienen las empresas de la región en el uso de las tecnologías sustentables dentro de sus propias instalaciones, se ha venido trabajando en un programa de participación escuela-empresa para que sean ellos el punto de partida en que sean base en la región para propiciar el cuidado al medio ambiente, con el cual ellos están comprometidos ya que sus certificaciones así lo prueban.

Tal vez, en un futuro un poco más lejano, se podrá proponer ante el Congreso del Estado la generación de una legislación que propicie el uso de las tecnologías sustentables en beneficio del medio ambiente a través de su uso en casas-habitación, primeramente, y posteriormente en empresas. 
Revista Iberoamericana

de las Ciencias Sociales y

Humanísticas

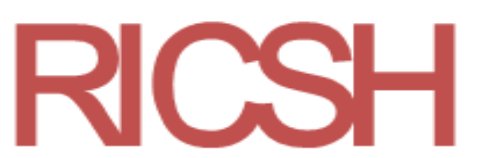

ISSN: 2395 - 7972

\section{Referencias}

Acciona. (2016). Energía solar fotovoltaica. Recuperado de https://www.acciona.com/es/energias-renovables/energia-solar/fotovoltaica/.

Baeza, M. (s. f.). Vivienda sustentable. Recuperado de http://www.senado.gob.mx/comisiones/vivienda/foros/ponencia_marcos_baeza.pdf.

Barnett, T. L. (23 de noviembre de 2017). Bio-Reconstruye México busca crear una "arquitectura para la vida" tras los sismos de septiembre. Archdaily. Recuperado de https://www.archdaily.mx/mx/tag/organi-k.

Bohoslavski, M. (1998). Las tecnologías alternativas. Madrid, España: Random House Mondadori.

Calvente, A. M. (2007). El concepto moderno de sustentabilidad. Recuperado de http://www.sustentabilidad.uai.edu.ar/pdf/sde/UAIS-SDS-100-002\%20$\% 20$ Sustentabilidad.pdf.

Core Writing Team, Pachauri, R. K. and Meyer, L.A. (eds.) (2014). Climate Change 2014: Synthesis Report. Contribution of Working Groups I, II and III to the Fifth Assessment Report of the Intergovernmental Panel on Climate Change. Geneva, Switzerland: Intergovernmental Panel on Climate Change.

EcoInventos. (2019). Construcción de recolectores de aguas pluviales. EcoInventos Green Technology. Recuperado de https://ecoinventos.com/.

Fehrenbacher, K. (January 12, 2016). U.S Solar Jobs Boom While Oil, Coal Struggle. Fortune. Retrieved from https://fortune.com/2016/01/12/solar-jobs-boom/.

Fredrick, H. (20 de noviembre de 2017). Ventajas y desventajas de los inodoros de doble descarga. enow en Español. Recuperado de https://www.ehowenespanol.com/ventajas-desventajas-inodoros-doble-descargainfo_298890/.

Garrett, C. (27 de mayo de 2020). Huella ecológica: definición, cálculo y reducción. Selectra. Recuperado de https://climate.selectra.com/es/que-es/huella-ecologica.

La Silla Rota. (22 marzo de 2018). El consumo promedio de agua en México. La Silla Rota. Recuperado de https://lasillarota.com/consumo-pomedio-agua-mexico/212034.

Miceli, A. (2016). Ecotecnias. Revista Vivienda, (646), 70-78.

Organización de las Naciones Unidas [ONU]. (1987). Informe de la Comisión Mundial sobre el Medio Ambiente y el Desarrollo. Nuestro futuro común. Oxford University Press. 
Revista lberoamericana

de las Ciencias Sociales y Humanísticas

ISSN: 2395 - 7972

Recuperado de https://sswm.info/sites/default/files/reference_attachments/UN\%20WCED\%201987 \%20Brundtland\%20Report.pdf.

Ral Aluminio. (s. f.). ¿Qué es un doble vidrio o DVH? Recuperado de https://ralaluminio.com.ar/que-es-un-doble-vidrio-o-dvh/.

Santamaria, P. (17 de octubre 2012). ¿Qué es la iluminación LED? Especial: Iluminación LED. Xataka smart home. Recuperado de https://www.xatakahome.com/iluminacion-y-energia/que-es-la-iluminacion-ledespecial-iluminacion-led. 